\title{
A DATA-DRIVEN METHOD FOR THE STEADY STATE OF RANDOMLY PERTURBED DYNAMICS
}

\author{
YAO LI
}

\begin{abstract}
We demonstrate a data-driven method to solve for the invariant probability density function of a randomly perturbed dynamical system. The key idea is to replace the boundary condition of numerical schemes by a least squares problem corresponding to a reference solution, which is generated by Monte Carlo simulation. With this method we can solve for the invariant probability density function in any local area with high accuracy, regardless of whether the attractor is covered by the numerical domain.
\end{abstract}

\section{INTRODUCTION}

Many physical and biological systems are subject to random perturbations. The time evolution of the probability density function of a randomly perturbed dynamical system, i.e., a stochastic differential equation, is usually described by the FokkerPlanck equation [28]. In many studies, the invariant probability density function of the randomly perturbed system is particularly important. It is well known that under suitable conditions, the invariant probability density function solves the steady state Fokker-Planck equation. On the other hand, the positive solution to the steady state Fokker-Planck equation must be an invariant probability density function of the corresponding stochastic differential equation [6, 5, 16].

When the unperturbed dynamical system has complex dynamics, an analytical solution of the Fokker-Planck equation is usually not available. On the other hand, numerically solving the steady state Fokker-Planck equation on an unbounded domain is often challenging due to the lack of a well-posed boundary condition. The usual practice is to let the numerical domain cover the global attractor of the unperturbed dynamical system with sufficient margin. The Freidlin-Wentzell theory [11] guarantees that the invariant probability density is close to zero when sufficiently far away from the global attractor. Then it is usually safe to assume a zero boundary condition.

The resolution of the numerical solution imposes additional challenges. When the strength of a random perturbation is $0<\sigma \ll 1$, it is known that the probability density function should concentrate on a $O(\sigma)$-neighborhood of the attractor [21]. Hence the grid size of the discretization cannot be larger than $\sigma$. Otherwise the numerical scheme cannot "see" the concentration, and sometimes serious numerical artifacts may occur. Therefore, when the noise strength is small and the underlying dynamical system has complex (possibly chaotic) dynamics, the grid size of the discretization has to be sufficiently small. In addition, chaos only occurs in ordinary differential equations in dimension $\geq 3$. This makes a numerical study of interplays 
between chaos and random perturbations extremely difficult. Take the Lorenz system for an example. A very expensive numerical computation in [2] can only solve the Fokker-Planck equation corresponding to the Lorenz system on a $160 \times 160 \times 160$ mesh, with a grid size $\approx 0.3$.

The boundary condition is not a problem any more if one uses Monte Carlo simulations to compute the invariant probability density function. A Monte Carlo simulation either runs the stochastic differential equation for a long time, or runs many independent trajectories of the stochastic differential equation for a finite amount of time. The Monte Carlo simulation is an efficient way to obtain statistics such as the expectation of a certain observable. However, the classical Monte Carlo simulation has severe accuracy problem when solving for the invariant probability density function. Unless one can generate a huge amount of samples, the probability density function generated by Monte Carlo simulation is usually too "noisy" to be useful.

In this paper we present a hybrid method that bypasses the disadvantages of classical numerical PDE approach and the Monte Carlo simulation. The key idea is to work on a domain without using any boundary conditions. Then the discretization of a steady state Fokker-Planck equation becomes underdetermined, which essentially gives a linear constraint. Instead of the boundary condition, we generate an approximated invariant probability density function by using the Monte Carlo simulation. This approximated density function does not have to be very accurate, because it only serves as a reference of the next step. Finally, we solve a least squares optimization problem under the linear constraint given by the discretization. The resultant solution satisfies the discretization of the steady state Fokker-Planck equation (without a boundary condition), and has minimum $L^{2}$ distance to the approximated density function generated by the Monte Carlo simulation. The least squares problem is further converted to a linear system that can be solved either exactly or using iterative methods. This method can help us to compute a high resolution solution in a local area without worrying about boundary conditions.

We demonstrate several numerical examples in this paper. The 1D double-well potential is used to test the accuracy and performance of the algorithm. The overall accuracy is satisfactory considering the performance. Then we demonstrate the strength of this method with $2 \mathrm{D}$ and $3 \mathrm{D}$ examples. In the $2 \mathrm{D}$ example, we show that a transition from relaxation oscillations to a smaller limit cycle is destroyed by small noise. A local solution with high resolution is presented to demonstrate some interesting local structures in the invariant probability density function. In 3D examples, we compute invariant probability density functions of small random perturbations of two chaotic oscillators, the Lorenz oscillator and the Rössler oscillator. With low computation cost, we are able to find numerical solutions with much higher resolution (grid size $=0.05$ ) than in previous studies.

We remark that the purpose of this paper is only to introduce a general framework. The detailed implementation can be further improved in many ways. For example, a divide-and-conquer strategy can significantly improve the performance 
of this hybrid algorithm. The naive Monte Carlo simulation can be replaced by various importance sampling techniques [29, 32]. If the noise is large enough to smear fine structures, the high dimensional Monte Carlo sampler proposed in [8, 9] can be adopted to our framework. The finite difference discretization can be replaced by other advanced solvers like the finite element method or other methods for high dimensional problems [26, 31, 33]. We will write several subsequent papers to address these issues.

\section{Probability AND NUMERICS PRELIMINARY}

2.1. Problem setting. Consider an autonomous ordinary differential equation

$$
x^{\prime}=f(x), \quad x \in \mathbb{R}^{n} .
$$

We are particularly interested in situations when equation 2.1) generates non-trivial dynamics. For example, equation (2.1) may admit a strange attractor or have separation of time scales that leads to interesting dynamics like the folding singularity, mixed mode oscillations etc. [14, 10].

Now we consider the following dynamical system with random perturbations, i.e., a stochastic differential equation (SDE),

$$
\mathrm{d} X_{t}=f\left(X_{t}\right) \mathrm{d} t+\sigma\left(X_{t}\right) \mathrm{d} W_{t},
$$

where $X_{t} \in \mathbb{R}^{n}, f: \mathbb{R}^{n} \rightarrow \mathbb{R}^{n}$ is a vector field, $\sigma: \mathbb{R}^{n} \rightarrow \mathbb{R}^{n \times n}$ is a matrix-valued function, and $\mathrm{d} W_{t}$ is the $n$-dimensional white noise. Throughout this paper, we assume that equation (2.2) admits a unique diffusion process solution $X_{t}$. (See $(\mathbf{H})$ below for the full assumption.) Note that the existence and the uniqueness of $X_{t}$ follow from mild assumptions on $f$ and $\sigma$, e.g., Lipschitz continuity of $f$ and $\sigma$ [27, 18].

Since $X_{t}$ is a diffusion process, we denote the transition kernel of $X_{t}$ by $P^{t}(x, A)=$ $\mathbb{P}\left[X_{t} \in A \mid X_{0}=x\right]$. A probability measure $\pi$ is said to be invariant if $\pi P^{t}=\pi$, where the left operator is defined as

$$
\pi P^{t}(A)=\int_{\mathbb{R}^{n}} P^{t}(x, A) \pi(\mathrm{d} x) .
$$

It is well known that the time evolution of the probability density function of $X_{t}$, denoted by $u_{t}$, is described by the Fokker-Planck equation

$$
u_{t}=\mathcal{L} u=-\sum_{i=1}^{n}\left(f_{i} u\right)_{x_{i}}+\frac{1}{2} \sum_{i, j=1}^{n}\left(D_{i, j} u\right)_{x_{i} x_{j}}, \quad u(0, x)=u_{0}(x),
$$

where $D=\sigma^{T} \sigma, u_{0}(x)$ is the probability density function of $X_{0}$. A probability density function $u_{*}(x)$ is said to be an invariant probability density function if $\mathcal{L} u_{*}=$ 0 . It is easy to see that an invariant probability density function defines an invariant probability measure $\pi$ of $X_{t}$, and $u_{*}(x)$ is the probability density function of $\pi$.

The existence of an invariant probability measure is guaranteed if $X_{t}$ is defined on a compact manifold without boundary [34. When $X_{t}$ is defined on unbounded domain, such existence needs some "dissipation" conditions [4, 16, 19]. The uniqueness of $\pi$ usually follows if $D$ is non-degenerate (everywhere positive definite). The 
convergence to the invariant probability measure is another tricky issue. To make $P^{t}(x, \cdot) \rightarrow \pi$ as $t \rightarrow \infty$, one needs stronger "dissipation" conditions and some minorization-type conditions [25, 15, 19]. Since the theme of this paper is to introduce a numerical algorithm, we have the following assumption on $X_{t}, P^{t}(x, \cdot)$, and $\pi$ throughout the paper.

(H) Equation (2.2) admits a unique diffusion process $X_{t}$. The diffusion process $X_{t}$ has a unique invariant probability measure $\pi$ that is absolutely continuous with respect to the Lebesgue measure. The probability density function of $\pi$ uniquely solves the stationary Fokker-Planck equation. In addition, $P^{t}(x, \cdot) \rightarrow \pi$ as $t \rightarrow \infty$ for every $x \in \mathbb{R}^{n}$.

2.2. Numerical PDE approach for computing invariant measure. There are two different approaches for computing $u_{*}$. One can either solve the Fokker-Planck equation (2.3) up to a sufficiently large $t$, or solve the stationary Fokker-Planck equation directly. The biggest problem of the numerical PDE approach is the boundary condition. For the sake of simplicity, we illustrate the problem by using the finite difference scheme. The case of the finite element scheme is analogous.

Without loss of generality, we solve the Fokker-Planck equation numerically on a 2D domain $[-L, L]^{2}$. Let the spatial and time step sizes be $r=2 L / N$ and $h$, respectively. Let $\mathbf{u}^{m}=\left\{u_{i, j}^{m}\right\}_{i, j=0}^{N}$ be the discretized solution at time step $m$. Entry $u_{i, j}^{m}$ is a numerical approximation of $u(m h, r i-L, r j-L)$. We need boundary conditions to update the solution to $\mathbf{u}^{m+1}$. The usual approach is to let the domain cover the global attractor of the ODE (2.1) with sufficient margin. Then we assume zero boundary conditions $u_{0, j}^{m}=u_{N, j}^{m}=u_{i, 0}^{m}=u_{i, N}^{m}=0$ and compute $\mathbf{u}^{m+1}$ by using either implicit Euler scheme or Crank-Nickson scheme. Since the probability of $X_{t} \notin[-L, L]^{2}$ is nonzero, $\mathbf{u}^{m}$ needs to be renormalized after each update such that

$$
\sum_{i, j=0}^{N} u_{i, j}^{m}=\frac{1}{r^{2}} .
$$

When $\left\|\mathbf{u}^{m}-\mathbf{u}^{m-1}\right\|$ is smaller than the error tolerance $\epsilon$ for some $m=M$, the update is stopped. Now $\mathbf{u}^{M}$ numerically solves the steady state Fokker-Planck equation. In order to make the numerical solution reliable, the domain has to be sufficiently large such that $\mathbb{P}\left[X_{t} \notin[-L, L]^{2}\right] \ll 1$ for $t \in[0, M h]$ and $1-\pi\left([-L, L]^{2}\right) \ll 1$.

Another approach is to solve the steady state Fokker-Planck equation directly. Assume the same 2D domain as before. In order to discretize $\mathcal{L} u_{*}$, the boundary value of $u_{*}$ on $\partial[-L, L]^{2}$ is necessary. The usual practice is to make $L$ large enough so that $[-L, L]^{2}$ covers the global attractor of equation (2.1) with sufficient margin. Then we can assume a zero boundary condition because of the Freidlin-Wentzell theory [11]. This will generate a linear system

$$
A \mathbf{u}=\mathbf{0},
$$

where $A$ is an $n \times n$ nonsingular matrix. To avoid the trivial solution, one also needs the constraint

$$
\mathbf{1}^{T} \mathbf{u}=r^{-2}
$$


This gives an overdetermined linear system

$$
\begin{aligned}
A \mathbf{u} & =\mathbf{0} \\
\mathbf{1}^{T} \mathbf{u} & =r^{-2} .
\end{aligned}
$$

Let

$$
\hat{A}=\left[\begin{array}{c}
A \\
\mathbf{1}^{T}
\end{array}\right] \quad, \quad \mathbf{b}=\left[\begin{array}{c}
\mathbf{0} \\
r^{-2}
\end{array}\right] .
$$

We can find the least squares solution $\hat{\mathbf{u}}$ that solves the optimization problem

$$
\min \|\hat{A} \hat{\mathbf{u}}-\mathbf{b}\|_{2} \text {. }
$$

The least squares solution $\hat{\mathbf{u}}$ numerically solves the steady state Fokker-Planck equation.

2.3. Probabilistic approach for computing invariant measure. The numerical PDE approach works reasonably well for 1D and 2D problems. However, in higher dimension this approach becomes not practical. In particular, the domain has to be sufficiently large to cover the global attractor of equation (2.1) with enough margin. This imposes great difficulty to many practical problems. For example, if equation (2.1) is a Lorenz system, then we need a grid in a $50 \times 50 \times 50$ box to cover the attractor. (See section 4.3 for more discussion about the Lorenz system.)

An alternative approach is to use Monte Carlo simulation. One can collect samples of $X_{t}$ over a long trajectory in any dimension, although the accuracy of the Monte Carlo simulation suffers greatly from the curse-of-dimensionality. Let $h \ll 1$ be the step size. Let $X_{n}:=X_{n h}$ be the numerical time- $h$ sample chain produced by certain numerical method (Euler, Milstein, Runge-Kutta .etc) [20]. Under certain conditions, $X_{n}$ admits an invariant probability measure $\pi_{h}$ that converges to $\pi$ as $h \rightarrow 0$ [24, 23]. In addition, $X_{n}$ is a Markov chain. Let $\xi: \mathbb{R}^{n} \rightarrow \mathbb{R}$ be an observable on $\mathbb{R}^{n}$ and $\mathbf{N}$ be the number of samples. By the law of large numbers of Markov chains [25], we have

$$
\frac{1}{\mathbf{N}} \sum_{n=1}^{\mathbf{N}} \xi\left(X_{n}\right) \rightarrow \pi(\xi) \quad \text { a.s. . }
$$

Therefore, we can use Monte Carlo simulation to compute the probability density function of $\pi$. For the sake of simplicity we consider grid points $\mathbf{u}=\left\{u_{i, j}\right\}_{i, j=0}^{N}$ in a $2 \mathrm{D}$ domain $[-L, L]^{2}$, such that $u_{i, j}$ is the numerical approximation of $u_{*}(i r-L, j r-$ $L)$. Let $O_{i, j}=[i r-L-r / 2, i r-L+r / 2] \times[j r-L-r / 2, j r-L+r / 2]$. Then the Monte Carlo simulation gives

$$
u_{i, j}=\frac{1}{\mathbf{N} r^{2}} \sum_{n=1}^{N} \mathbf{1}_{O_{i, j}}\left(X_{n}\right)
$$

for some sufficiently large $\mathbf{N}$. In practice, we construct $(N+1)^{2}$ boxes $O_{i, j}$ and simulate $X_{n}$ over a long time period. After the simulation, $u_{i, j}$ is obtained by counting sample points of $X_{n}$ falling into $O_{i, j}$. 
It is easy to see that the Monte Carlo simulation approach has a significant disadvantage on the accuracy because it is difficult to collect enough sample points in each $O_{i, j}$. Without loss of generality, we assume

$$
\sum_{n=1}^{\mathbf{N}} \mathbf{1}_{O_{i, j}}\left(X_{n}\right)=O\left(r^{2} \mathbf{N}\right) .
$$

If we treat $\mathbf{1}_{O_{i, j}}\left(X_{n}\right)$ as i.i.d Bernoulli random variables, some calculation shows that the standard deviation of $u_{i, j}$ is $O\left(r^{-1} \mathbf{N}^{-1 / 2}\right)$. Hence $\mathbf{N}$ has to be very large to control the standard deviation. For example, $\mathbf{N}$ needs to be $O\left(r^{-5}\right)$ to reduce the standard deviation to $O\left(r^{2}\right)$. The accuracy problem will be much worse in higher dimensions. In practice, the solution obtained from the Monte Carlo simulation usually looks very "noisy".

Despite of its accuracy problem, the Monte Carlo simulation has more flexibility because of the following reasons. (1) In the Monte Carlo simulation, the domain $[-L, L]^{2}$ does not have to cover the global attractor of (2.1). (2) The curse of dimensionality is slightly alleviated if equation (2.1) has a lower dimensional global attractor. Because the invariant probability measure $\pi$ concentrates on the vicinity of the global attractor of equation (2.1). (3) Parallel computing is much easier for Monte Carlo simulations. (4) Some high dimensional sampling technique can be applied to improve the Monte Carlo simulation for a large class of dynamical systems.

\section{A HYBRID DATA-DRIVEN METHOD}

We propose the following hybrid method that combines the high accuracy of the numerical PDE approach and the flexibility of the Monte Carlo simulation. Consider a $2 \mathrm{D}$ domain $\left[a_{0}, b_{0}\right] \times\left[a_{1}, b_{1}\right]$ that does not have to cover any attractor of equation (2.1). Let $\mathbf{u}=\left\{u_{i, j}\right\}_{i=1, j=1}^{i=N, j=M}$ be the numerical solution. Without loss of generality assume $r=\left(b_{0}-a_{0}\right) / N=\left(b_{1}-a_{1}\right) / M$. Then $u_{i, j}$ approximates $u_{*}$ at the grid point $\left(i r+a_{0}, j r+a_{1}\right)$. By discretizing the steady state Fokker-Planck equation $\mathcal{L} u_{*}=0$ without any boundary condition, we have a linear system with normalizing condition

$$
\left\{\begin{array}{ccc}
B \mathbf{u} & = & 0 \\
\mathbf{1}^{T} \mathbf{u} & = & r^{-2}
\end{array} .\right.
$$

Let

$$
\hat{B}=\left[\begin{array}{c}
B \\
\mathbf{1}^{T}
\end{array}\right] \quad, \quad \mathbf{b}=\left[\begin{array}{c}
\mathbf{0} \\
r^{-2}
\end{array}\right] .
$$

Obviously $\hat{B}$ is not a full-rank matrix. Hence we obtain a linear constraint $\hat{B} \mathbf{u}=\mathbf{b}$.

Then we run the Monte Carlo simulation to get another approximate solution $\mathbf{v}=\left\{v_{i, j}\right\}_{i=1, j=1}^{i=N, j=M}$. Let $O_{i, j}=\left[a_{0}+i r-r / 2, a_{0}+i r+r / 2\right] \times\left[a_{1}+j r-r / 2, a_{1}+j r+r / 2\right]$. Let $\mathbf{N}$ be a large number, the Monte Carlo simulation gives

$$
v_{i, j}=\frac{1}{\mathbf{N} r^{2}} \sum_{n=1}^{N} \mathbf{1}_{O_{i, j}}\left(X_{n}\right) .
$$


The approximate solution $\mathbf{v}$ in this step does not have to be very accurate. We will use it in the next step to obtain a much more accurate numerical solution $\mathbf{u}$.

The key step of this hybrid solution is to solve the following optimization problem. We combine the linear constraint with the "noisy" data $\mathbf{v}$ obtained from the Monte Carlo simulation. The idea is that $\mathbf{u}$ should both satisfy the linear constraint from the discretization and be as close to $\mathbf{v}$ as possible. This leads to the optimization problem

$$
\begin{array}{cl}
\min & \|\mathbf{u}-\mathbf{v}\|_{2} \\
\text { subject to } & \hat{B} \mathbf{u}=\mathbf{b} .
\end{array}
$$

Let $\mathbf{x}=\mathbf{u}-\mathbf{v}$, this reduces to the problem

$$
\begin{array}{cl}
\min & \|x\|_{2} \\
\text { subject to } & \hat{B} \mathbf{x}=\mathbf{d},
\end{array}
$$

where

$$
\mathbf{d}=\mathbf{b}-\hat{B} \mathbf{v} .
$$

The following theorem is a straightforward textbook result. (See for example [7].) We include the proof of the sake of completeness of the paper.

Theorem 3.1. If $\hat{B}$ has linearly independent rows, then

$$
\hat{\mathbf{x}}=\hat{B}^{T}\left(\hat{B} \hat{B}^{T}\right)^{-1} \mathbf{d}
$$

is the unique solution of 3.2 .

Proof. It is easy to see that $\hat{\mathbf{x}}$ solves the linear constraint $\hat{B} \hat{\mathbf{x}}=\mathbf{d}$.

For any vector $\mathbf{x} \neq \hat{\mathbf{x}}$ satisfying $\hat{B} \hat{\mathbf{x}}=\mathbf{d}$, we have

$$
\begin{aligned}
\|\mathbf{x}\|^{2} & =\|\hat{\mathbf{x}}+\mathbf{x}-\hat{\mathbf{x}}\|^{2} \\
& =\|\hat{\mathbf{x}}\|^{2}+2 \hat{\mathbf{x}}^{T}(\mathbf{x}-\hat{\mathbf{x}})+\|\mathbf{x}-\hat{\mathbf{x}}\|^{2} .
\end{aligned}
$$

Since $\hat{B} \mathbf{x}=\mathbf{d}$, we have

$$
\hat{\mathbf{x}}^{T}(\mathbf{x}-\hat{\mathbf{x}})=\mathbf{d}^{T}\left(\hat{B}^{T} \hat{B}\right)^{-1} \hat{B}(\mathbf{x}-\hat{\mathbf{x}})=0 .
$$

Therefore, we have

$$
\|\mathbf{x}\|^{2}=\|\hat{\mathbf{x}}\|^{2}+\|\mathbf{x}-\hat{\mathbf{x}}\|^{2} \geq\|\hat{\mathbf{x}}\|^{2} .
$$

This completes the proof.

An efficient way to solve equation $(3.3)$ is to use the $\mathrm{QR}$ factorization $\hat{B}^{T}=Q R$. After a QR factorization, we have

$$
\hat{\mathbf{x}}=Q\left(R^{-1}\right)^{T} \mathbf{d} .
$$

Then $\mathbf{u}=\hat{\mathbf{x}}+\mathbf{v}$ is the desired numerical solution.

Heuristically, it is easy to see that the optimization problem (3.1) can significantly reduce the error of the data $\mathbf{v}$ generated by the Monte Carlo simulation. Assume the Monte Carlo sampler does not have significant bias. Let $\mathbf{u}_{*}=\left\{u_{*}^{i, j}\right\}_{i=1, j=1}^{i=N, j=M}$ be the vector corresponding to the exact solution, i.e., $u_{*}^{i, j}=u_{*}\left(i r+a_{0}, j r+b_{0}\right)$. Then the error term $\mathbf{w}:=\mathbf{v}-\mathbf{u}_{*}$ can be approximated by a random vector such that 
each entry has zero expectation and finite variance. Let $\hat{\mathbf{u}}$ be the solution to the optimization problem (3.1). Then the new error term $\hat{\mathbf{w}}:=\hat{\mathbf{u}}-\mathbf{u}_{*}$ is approximated by $\mathcal{P}\left(\mathbf{v}-\mathbf{u}_{*}\right)$, where $\mathcal{P}$ is the projection operator corresponding to the hyperplane given by $\hat{B} \mathbf{u}=\mathbf{b}$. With high probability, the projection of a random vector $\mathbf{w}$ to a much lower dimensional hyperplane has much smaller norm. In other words we have $\|\hat{\mathbf{w}}\| \ll\|\mathbf{w}\|$ with high probability. The full proof is much longer than the above heuristic description. Since the aim of this paper is to introduce the algorithm, we prefer to put the rigorous proof about this algorithm into our subsequent paper.

Finally, we remark that one significant advantage of this approach is that we can obtain a high resolution solution in any local area. There is no restriction on the domain as long as the Monte Carlo simulation can produce enough sample points. If a global solution is necessary, we can divide the space into many subdomains $I_{1}, \cdots, I_{K}$, solve them separately, and combine them together according to the Monte Carlo simulation. (The probability of a subdomain $\pi\left(I_{k}\right)$ can be obtained from the Monte Carlo simulation, which is the weight of $I_{k}$ when generating the global solution.) This divide-and-conquer strategy allows us to solve large scale problems ( $\sim 10^{9}$ grid points) on a laptop. We will address it in full details in our subsequent paper.

\section{NumERICAL EXAMPLES}

We will illustrate our hybrid approach with three examples: the double well potential gradient flow, the Van der Pol oscillator, and 3D chaotic oscillators.

4.1. Double-well potential and error analysis. The first example is the gradient flow with respect to a double-well potential. Consider the potential function

$$
U=\frac{1}{2} x^{4}-x^{2}
$$

and the stochastic differential equation

$$
\mathrm{d} X_{t}=-U^{\prime}\left(X_{t}\right) \mathrm{d} t+\sigma \mathrm{d} W_{t}
$$

for $\sigma=0.6$. The probability density function of this system is

$$
u_{*}(x)=\frac{1}{K} e^{-2 U(x) / \sigma^{2}},
$$

where $K$ is a normalizer.

In this example, we demonstrate our method by solving for $u_{*}(x)$ on the interval of $[0,2]$. Note that this system has two equilibria at \pm 1 . Equilibrium $x=-1$ is not covered by the numerical domain $[0,2]$. In particular, we have $u_{*}(0)=0.1062$ so the zero boundary condition does not apply to this problem. We also show the accuracy and computational time of the hybrid method in this example.

Let $X_{n}$ be the discrete-time Markov chain given by the EulerMaruyama method with a fixed time step size $\mathrm{d} t=0.001$. The Monte Carlo simulation is done by simulating $X_{n}$ up to time $T=\mathrm{N} h$. The numerical solution from the optimization problem, the exact probability density function $u_{*}(x)$, and the approximate density function from Monte Carlo simulation are compared in Figure 1. We can see that 
the Monte Carlo simulation itself only produces a solution with low accuracy, unless one can collect a huge amount of samples. In fact, when $h=0.005, T$ needs to be at least $10^{5}$ to remove the sawtooth in the solution (the orange plot in Figure 1). The rough solution generated by the Monte Carlo simulation is smoothed and corrected by the linear constraint (the red plot in Figure 1).

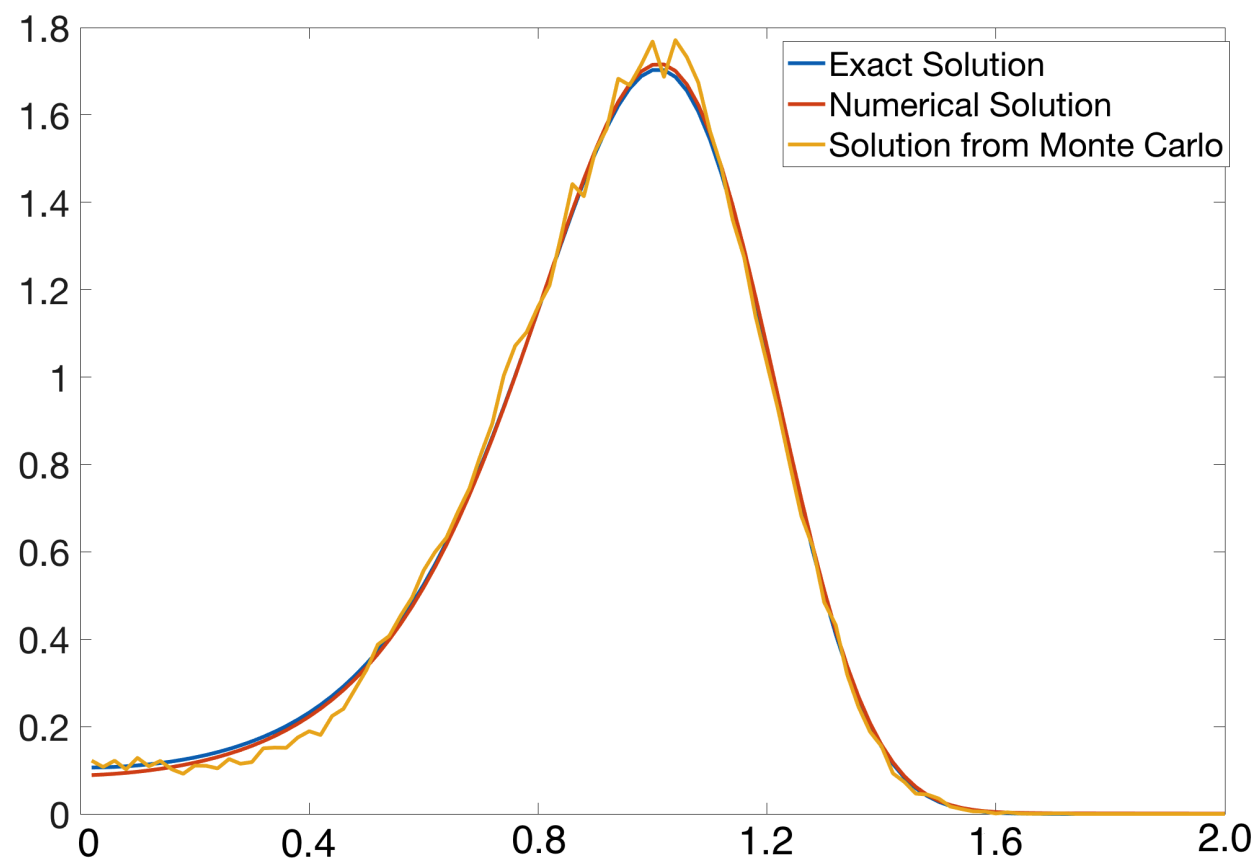

Figure 1. A comparison of exact solution, solution from Monte Carlo, and solution from the hybrid method.

The following table compares $L^{2}$ errors with varying time span $T$ and grid size $h$. Each entry is the average $L^{2}$ error of 5 trials. We can see that despite some randomness caused by the Monte Carlo simulation, the error drops with smaller grid size and larger sample size for the Monte Carlo simulation. Note that the invariant probability measure of $X_{n}$ is only an $O(\mathrm{~d} t)=1.0 \times 10^{-3}$ approximation of that of $X_{t}$. Hence it does not make sense to test the accuracy with more samples or smaller grid size unless one makes the time step even smaller. In this small-scale 1D problem, the classical numerical PDE solver is more accurate, mainly because the invariant probability measure of $X_{n}$ is only a first order approximation of that of $X_{t}$. But overall the accuracy is satisfactory given the performance of the algorithm.

It remains to comment on the computation time. We choose smaller grid sizes $h=2 \times 10^{-4}, 1 \times 10^{-4}$, and $5 \times 10^{-5}$ to highlight the difference between different approaches. The time span of Monte Carlo simulation is chosen to be $T=4000$. In order to apply the numerical PDE approach directly, one needs to enlarge the 


\begin{tabular}{|c|c|c|c|c|}
\hline$T \backslash h$ & 0.04 & 0.02 & 0.01 & 0.005 \\
\hline 500 & $6.233 \times 10^{-3}$ & $5.971 \times 10^{-3}$ & $2.260 \times 10^{-3}$ & $2.120 \times 10^{-3}$ \\
\hline 1000 & $4.289 \times 10^{-3}$ & $5.679 \times 10^{-3}$ & $2.137 \times 10^{-3}$ & $2.893 \times 10^{-3}$ \\
\hline 2000 & $2.240 \times 10^{-3}$ & $2.562 \times 10^{-3}$ & $2.483 \times 10^{-3}$ & $1.476 \times 10^{-3}$ \\
\hline 4000 & $1.914 \times 10^{-3}$ & $2.159 \times 10^{-3}$ & $1.339 \times 10^{-3}$ & $0.656 \times 10^{-3}$ \\
\hline
\end{tabular}

TABLE 1. Accuracy of the hybrid method with respect to different sample sizes and grid sizes.

\begin{tabular}{|c|c|c|c|c|}
\hline grid size $h$ & Numerical PDE & Hybrid (Total) & Hybrid (phase 1) & Hybrid (phase 2) \\
\hline $2 \times 10^{-4}$ & $0.05943 \mathrm{sec}$ & $0.2775 \mathrm{sec}$ & $0.2771 \mathrm{sec}$ & $0.004236 \mathrm{sec}$ \\
\hline $1 \times 10^{-4}$ & $0.2565 \mathrm{sec}$ & $0.2668 \mathrm{sec}$ & $0.2663 \mathrm{sec}$ & $0.004887 \mathrm{sec}$ \\
\hline $5 \times 10^{-5}$ & $1.111 \mathrm{sec}$ & $0.2618 \mathrm{sec}$ & $0.2609 \mathrm{sec}$ & $0.009218 \mathrm{sec}$ \\
\hline
\end{tabular}

TABLE 2. Computational time for the numerical PDE approach and the hybrid method.

domain to $[-2,2]$ to apply the zero boundary condition. The direct Monte Carlo simulation is too slow to be interesting if one wants to achieve the same accuracy. Hence we only compare the numerical PDE approach and the hybrid method in the following table. The computation time for the hybrid method is further broken down in to the Monte Carlo simulation phase (Phase 1) and the optimization phase (Phase 2). The numerical PDE approach uses the MATLAB solver mldivide (the backslash solver). The Monte Carlo simulation is written in $\mathrm{C}++$. The optimization problem equation (3.1) is solved by the MATLAB solver lsqminnorm.

From Table 2, we can find that solving the optimization problem (3.1) is actually much faster than solving the least squares problem (2.4). This is because the numerical PDE approach has to cover both equilibria with considerable margin in order to apply the zero boundary condition. In addition, solving the overdetermined least squares problem in equation (2.4) takes more time for the MATLAB solver we use. In this problem, the Monte Carlo simulation is the bottleneck of the hybrid method. In higher dimensional problems, such as the Lorenz oscillator in Section 4.3, solving the optimization problem (3.1) usually takes much more time than the Monte Carlo simulation. And the classical numerical PDE approach becomes not practical any more, because the domain still has to cover the entire attractor in order to apply the zero boundary condition.

4.2. Van der Pol oscillator and canard. The second example is a Van der Pol oscillator, which has been intensively used in both physics and mathematical biology [13, 17]. In this subsection, we use our method to demonstrate an interesting phenomenon related to the canard solution. 
Consider an oscillator

$$
\begin{aligned}
\dot{x} & =\frac{1}{\epsilon}\left(y-\frac{1}{3} x^{3}+x\right) \\
\dot{y} & =a-x,
\end{aligned}
$$

where $\epsilon=0.1$ is the time scale separation parameter, and $a$ is a control parameter. This system is a prototypical example for the canard explosion. A "canard" is a solution that the system can pass a bifurcation point of the critical manifold and follow the repelling part of the slow manifold for some amount of time [3]. Usually a canard solution only exists for a very small range of parameters.

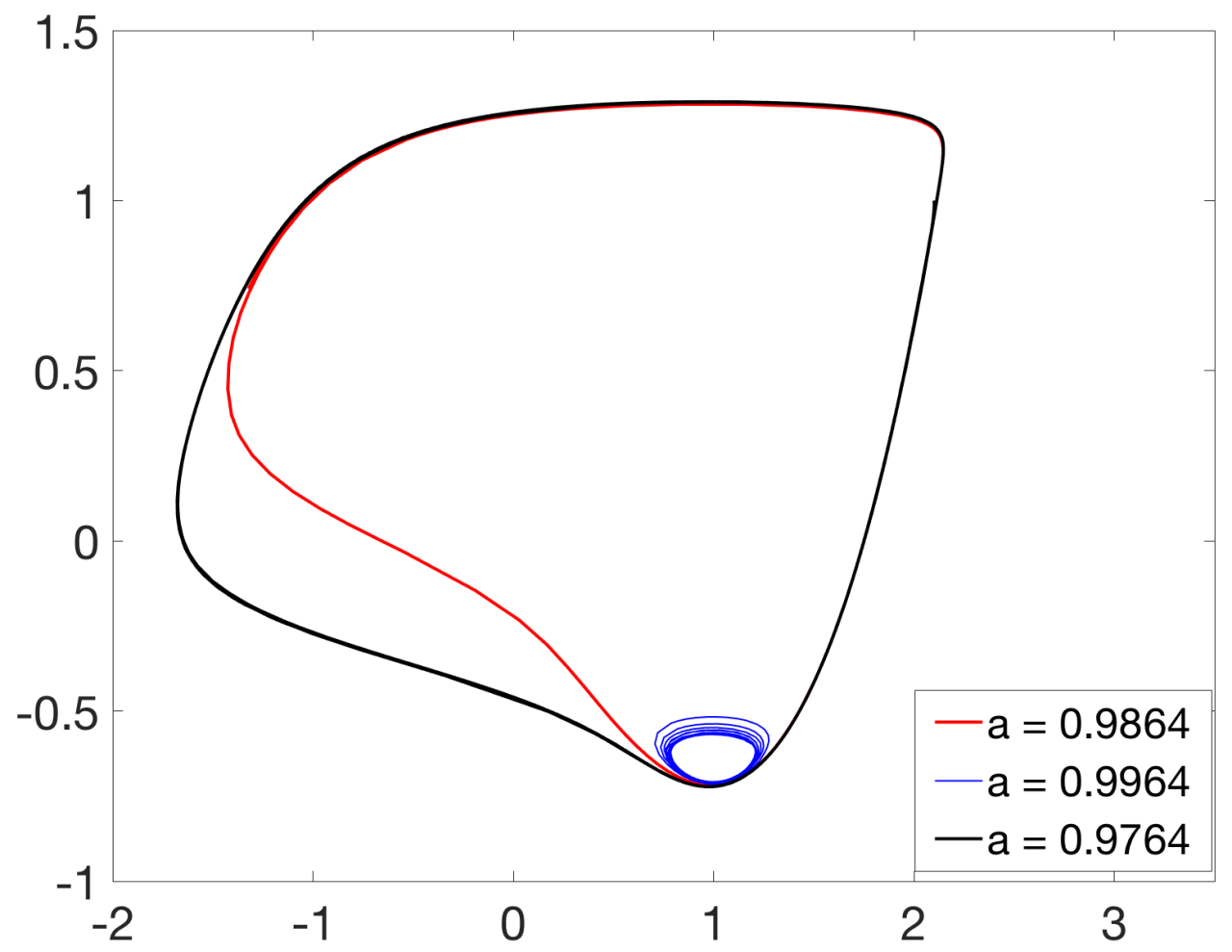

Figure 2. Bifurcation of the Van der Pol oscillator. When $a$ is small, the system demonstrates relaxation oscillations (Black). The red solution is a canard solution, at which the solution follows the repelling part of the slow manifold for a short period of time. With increasing $a$, a transition through the canard occurs and the solution follows a smaller limit cycle (Blue).

We consider the random perturbation of system 4.1

$$
\begin{aligned}
\mathrm{d} X_{t} & =\frac{1}{\epsilon}\left(Y_{t}-\frac{1}{3} X_{t}^{3}+X_{t}\right) \mathrm{d} t+\sigma \mathrm{d} W_{t}^{1} \\
\mathrm{~d} Y_{t} & =\left(a-X_{t}\right) \mathrm{d} t+\sigma \mathrm{d} W_{t}^{2},
\end{aligned}
$$


where $W_{t}^{1}$ and $W_{t}^{2}$ are two independent Wiener processes. The parameter $a$ is chosen to be 0.9964 , at which the deterministic system has already passed the canard bifurcation and is attracted to a smaller limit cycle (blue curve in Figure 2). We use our hybrid method to compute the density function $u_{*}(x)$ of the invariant probability measure of system (4.2). Our numerical result shows that this transition through a canard solution is essentially destroyed by a small random perturbation. Although the deterministic system admits a smaller limit cycle, the steady state probability density function $u_{*}(x)$ still concentrates on the large limit cycle corresponding to the relaxation oscillations (the black curve in Figure 2). When the strength of noise increases, the support of $u_{*}(x)$ not only becomes "wider", but also has significant deformation. When the noise is large $(\sigma=1.0)$, some probability density moves to the slow manifold that does not belong to any limit cycle of the deterministic system and forms two "tails". With the hybrid method introduced in this paper, we can get a high resolution local solution about the lower left "tail" with much higher precision (Panel 6 of Figure 3).

4.3. 3D chaotic oscillators under random perturbations. The hybrid method demonstrates its full strength in 3D systems. In this subsection, we compute numerical invariant probability density functions of two randomly perturbed chaotic systems: the Lorenz oscillator and the Rössler attractor. Both of them are typical chaotic oscillators that play a significant role in the study of nonlinear physics and dynamical systems [30, 12, 1, 22].

For the Lorenz system, we mean

$$
\begin{aligned}
\dot{x} & =a(y-x) \\
\dot{y} & =x(b-z)-y \\
\dot{z} & =x y-c z
\end{aligned}
$$

with typical parameters $a=10, b=28$, and $c=8 / 3$. It is well known that system (4.3) has a butterfly-shape strange attractor. The Rössler attractor is a chaotic oscillator that has the similar mechanism as the Lorenz oscillator. We have

$$
\begin{aligned}
\dot{x} & =-y-z \\
\dot{y} & =x+a y \\
\dot{z} & =b+z(x-c) .
\end{aligned}
$$

Again, we use typical parameters $a=0.2, b=0.2$, and $c=5.7$.

We are interested in invariant probability density functions of random perturbations of system (4.3) and system (4.4). In both systems, a perturbation term $\sigma \mathrm{d} \mathbf{W}_{t}$ is added to the deterministic part, where $\sigma>0$ is the strength of noise, and $\mathbf{W}_{t}$ is the standard Wiener process in $\mathbb{R}^{3}$. Needless to say, it is extremely difficult to solve a steady state Fokker-Planck equation in 3D on a large domain. Take the Lorenz oscillator as an example. If the numerical domain has to cover the attractor, we will solve a $3 \mathrm{D}$ equation on a cube $[-25,25] \times[-25,25] \times[0,50]$. When the grid size is 0.05 , the resultant numerical solution will have $10^{9}$ grid points. Solving such a large linear system is very computationally expensive. 

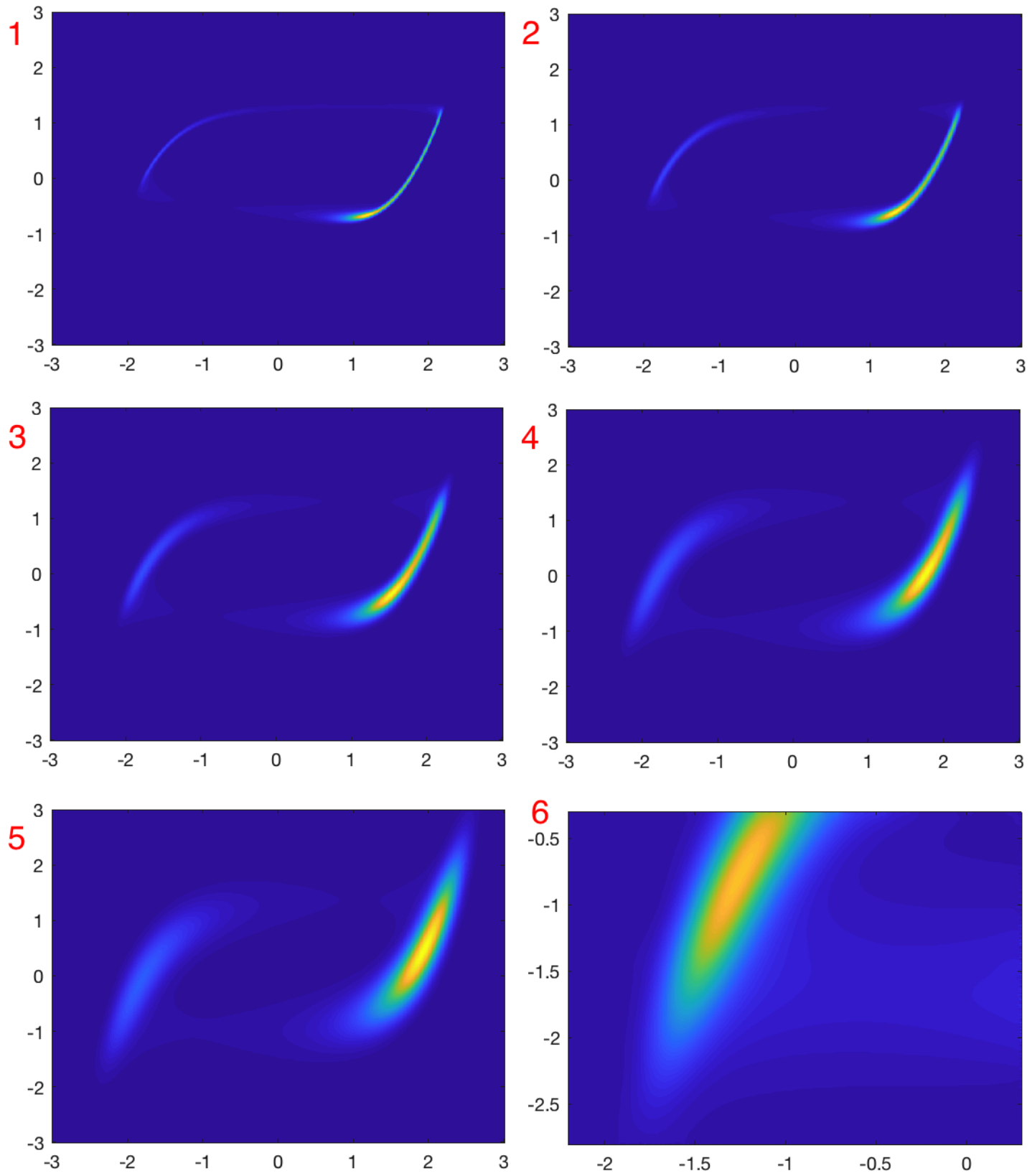

FIgURE 3. Numerical solutions of the invariant probability density function with different $\sigma$. Panel 1 to 6 : (1), $\sigma=0.1 ;(2), \sigma=0.2$; (3), $\sigma=0.4$; (4), $\sigma=0.7$; (5), $\sigma=1.0 ;$ (6) local solution of $\sigma=1.0$ showing the lower left "tail". 

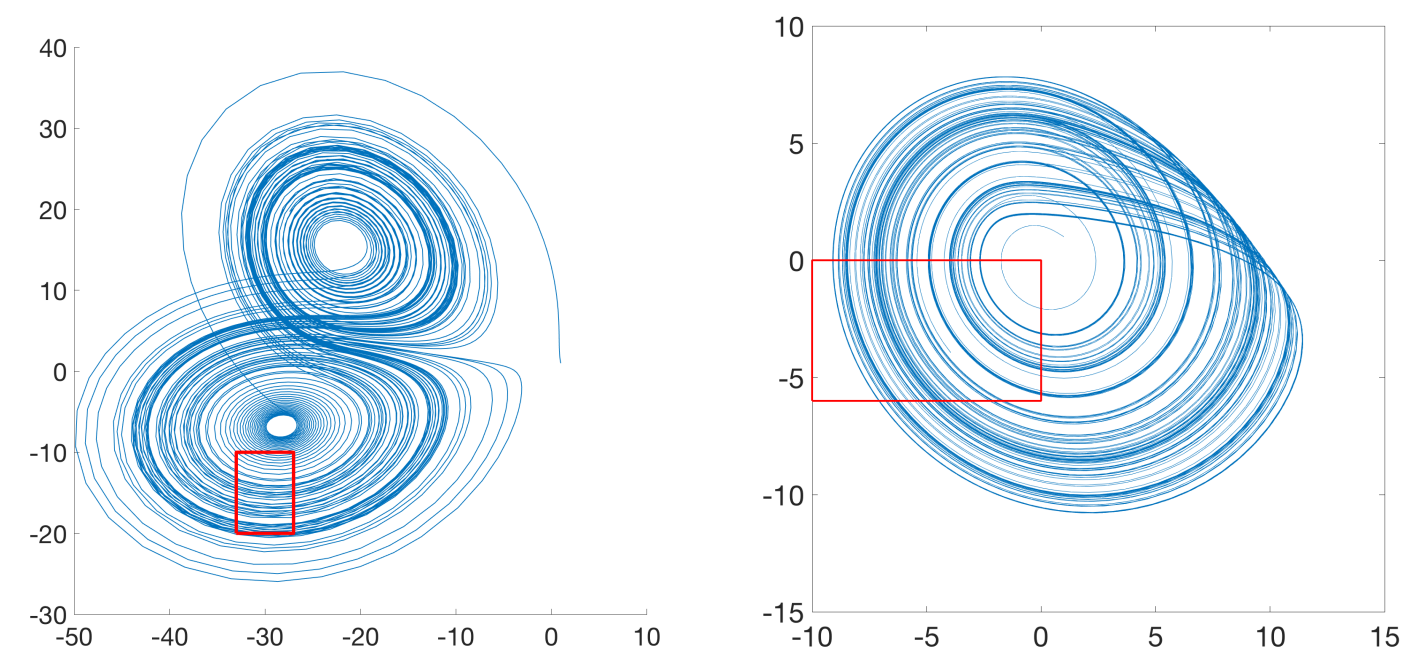

FigurE 4. Left: Trajectory of Lorenz oscillator. Right: Trajectory of the Rössler oscillator. Both: Solution is projected to the XY-plane. Red box is the domain of the numerical solution.

The hybrid method provides an approach to solve a 3D steady state Fokker-Planck equation locally with high resolution and low cost. If the global solution is still necessary, one can numerically solve many local solutions and "glue" them together. In Figure 4, we choose a small box on the attractor as the domain (the red rectangle). The attractor is projected to the XY-plane for the purpose of demonstration. Note that the Lorenz oscillator is rotated by a rotation matrix for the purpose of easier demonstration and mesh generation. The heights of both domains are 1.0. Then we use our hybrid method to compute the invariant probability density function. The strength of noise is chosen to be $\sigma=0.3$ for the Lorenz attractor and $\sigma=0.1$ for the Rössler attractor. We use larger $\sigma$ because the Lorenz system has a much bigger attractor. The grid size is 0.05 for both examples.

The numerical solution is demonstrated in Figure 5, in which the solution is integrated with respect to $z$ for the purpose of easier visualizations. We can see that both invariant probability density functions reveal lots of fine structures of the strange attractors. And the probability density is higher near the center of the attractor. In constrast to the very computationally expensive global problem, it only takes a laptop about 10 minutes to generate such a local solution on MATLAB.

\section{Conclusion}

In this paper we present a hybrid numerical method that solves the steady state Fokker-Planck equation. The numerical discretization scheme (finite difference scheme, finite element scheme, or Galerkin method) without boundary condition gives a linear constraint. A low-accuracy numerical solution produced by the Monte Carlo simulation (or other variants) serves as a reference solution. The problem is then converted to an optimization problem, which looks for the least squares solution with 

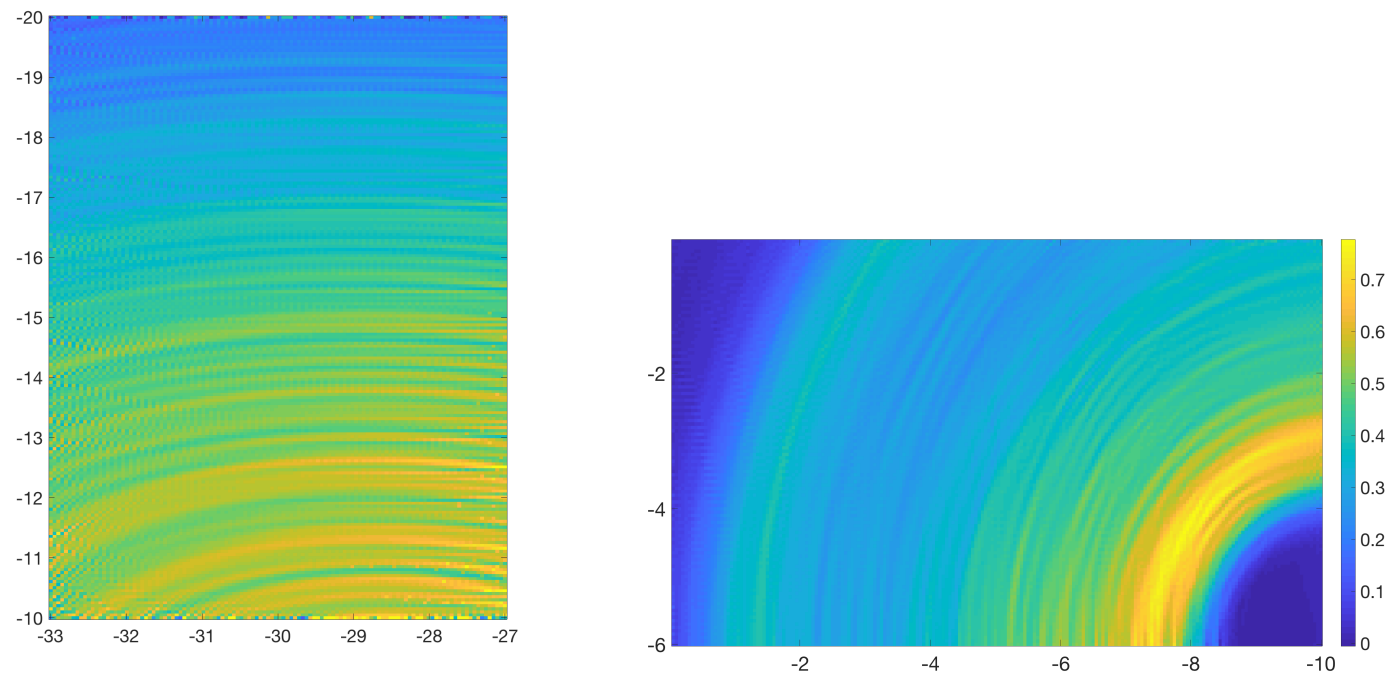

FIGURE 5. The invariant probability density function of the randomly perturbed system. Left panel: Invariant probability density function of the Lorenz oscillator with $\sigma=0.3$. Right panel: Invariant probability density function of the Rössler oscillator with $\sigma=0.1$. The numerical solution is projected to the XY-plane for the purpose of easier visualization.

respect to the reference solution, under the linear constraint given by the numerical discretization scheme.

The main advantage of this hybrid approach is that it drops the dependence on boundary conditions. Hence we can compute the steady state Fokker-Planck equation in any local area, while the traditional numerical PDE approach has to use a large enough domain in order to apply the zero boundary condition. This makes a significant difference if one wants to study the invariant probability density function in a local area in the vicinity of a strange attractor. The Monte Carlo simulation gives both good flexibility and some limitations to this hybrid method. Our simulation shows that the hybrid method can tolerate "local" fluctuations in the reference solution very well. However, if the Monte Carlo simulation result has significant and systemic bias, the hybrid method cannot completely recover the invariant probability density function. Also, the Monte Carlo simulation usually only generates very few samples (or no sample) in regions that are very far away from the attractor. In these regions, the hybrid method cannot recover the tail effectively, while the traditional PDE solver usually performs better.

This paper serves as the first paper of a series of investigations. Under this data-driven framework, lots of improvements can be made to both the Monte Carlo simulation and the numerical PDE approach. For example, because our data-driven framework does not rely on boundary conditions, one can use divide-and-conquer strategy to divide the domain into many "blocks". Our preliminary work shows 
that this approach can significantly accelerate the computation. Another potential improvement is to use some recently developed sampling techniques in the Monte Carlo simulation. In particular, if the noise term is large enough, the Gaussian mixture method reported in [8, 9] can significantly reduce the cost of Monte Carlo simulations for high-dimensional problems. We expect to incorporate this sampling technique into our framework in the future.

It remains to comment on the extension to the time dependent Fokker-Planck equation, as the time evolution of the probability density function is very importent in many applications. Our hybrid method can be extended to the time-dependent case after minor modifications. To use the hybrid method, at each time step, the classical PDE solver should be replaced by a least squares optimization problem. More precisely, let $\mathbf{u}_{n}$ denote the numerical solution to a time dependent FokkerPlanck equation at time step $n$. Then the numerical solution $\mathbf{u}_{n+1}$ at the next step is obtained by solving an optimization problem

$$
\begin{array}{cl}
\min & \left\|\mathbf{u}_{n+1}-\mathbf{v}_{n+1}\right\| \\
\text { subject to } & \mathbf{A} \mathbf{u}_{n+1}=\mathbf{b}_{n},
\end{array}
$$

where the linear contraint $\mathbf{A} \mathbf{u}_{n+1}=\mathbf{b}_{n}$ comes from the numerical discretization scheme (such as implicit Euler scheme or Crank-Nicolson scheme) for the time dependent Fokker-Planck equation, and $\mathbf{v}_{n+1}$ is a probability density function (with lower accuracy) generated by the Monte Carlo simulation.

\section{ACKNowledgement}

The author thanks his former students Ms. Lily Chou and Ms. Huangyi Shi for some preliminary numerical simulations related to this project.

\section{REFERENCES}

[1] Valentin S Afraimovich, VV Bykov, and Leonid P Shilnikov, On the origin and structure of the lorenz attractor, Akademiia Nauk SSSR Doklady, vol. 234, 1977, pp. 336-339.

[2] Altan Allawala and JB Marston, Statistics of the stochastically forced lorenz attractor by the fokker-planck equation and cumulant expansions, Physical Review E 94 (2016), no. 5, 052218.

[3] E Benoit, Canards et enlacements, Publications Mathématiques de l'Institut des Hautes Études Scientifiques 72 (1990), no. 1, 63-91.

[4] V. Bogachev and M. R "ockner, A generalization of khasminskii's theorem on the existence of invariant measures for locally integrable drifts, Theory of Probability and its Applications 45 (2001), 363.

[5] V.I. Bogachev, N.V. Krylov, and M. Röckner, Elliptic and parabolic equations for measures, Russian Mathematical Surveys 64 (2009), 973.

[6] VI Bogachev, M Röckner, and W Stannat, Uniqueness of invariant measures and maximal dissipativity of diffusion operators on l1, infinite dimensional stochastic analysis (proceedings of the colloquium, amsterdam, 11-12 february, 1999), ph, Clément, F. den Hollander, J. van Neerven and B. de Pagter eds., Royal Netherlands Academy of Arts and Sciences, 39-54.

[7] Stephen Boyd and Lieven Vandenberghe, Convex optimization, Cambridge university press, 2004.

[8] Nan Chen and Andrew J Majda, Beating the curse of dimension with accurate statistics for the fokker-planck equation in complex turbulent systems, Proceedings of the National Academy of Sciences 114 (2017), no. 49, 12864-12869. 
[9] _ Efficient statistically accurate algorithms for the fokker-planck equation in large dimensions, Journal of Computational Physics 354 (2018), 242-268.

[10] Mathieu Desroches, John Guckenheimer, Bernd Krauskopf, Christian Kuehn, Hinke M Osinga, and Martin Wechselberger, Mixed-mode oscillations with multiple time scales, Siam Review 54 (2012), no. 2, 211-288.

[11] Mark Iosifovich Freidlin and Alexander D Wentzell, Random perturbations, Random Perturbations of Dynamical Systems, Springer, 1998, pp. 15-43.

[12] Pierre Gaspard, Rössler systems, Encyclopedia of nonlinear science (2005), 808-811.

[13] John Guckenheimer, Dynamics of the van der pol equation, IEEE Transactions on Circuits and Systems 27 (1980), no. 11, 983-989.

[14] John Guckenheimer and Radu Haiduc, Canards at folded nodes, Moscow Mathematical Journal 5 (2005), no. 1, 91-103.

[15] Martin Hairer and Jonathan C Mattingly, Ergodicity of the 2d navier-stokes equations with degenerate stochastic forcing, Annals of Mathematics (2006), 993-1032.

[16] Wen Huang, Min Ji, Zhenxin Liu, and Yingfei Yi, Steady states of fokker-planck equations: I. existence, Journal of Dynamics and Differential Equations 27 (2015), no. 3-4, 721-742.

[17] Takashi Kanamaru, Van der pol oscillator, Scholarpedia 2 (2007), no. 1, 2202.

[18] Ioannis Karatzas and Steven Shreve, Brownian motion and stochastic calculus, vol. 113, Springer Science \& Business Media, 2012.

[19] Rafail Khasminskii, Stochastic stability of differential equations, vol. 66, Springer Science \& Business Media, 2011.

[20] Peter E Kloeden and Eckhard Platen, Numerical solution of stochastic differential equations, vol. 23, Springer Science \& Business Media, 2013.

[21] Yao Li and Yingfei Yi, Systematic measures of biological networks i: Invariant measures and entropy, Communications on Pure and Applied Mathematics 69 (2016), no. 9, 1777-1811.

[22] Edward N Lorenz, Deterministic nonperiodic flow, Journal of the atmospheric sciences 20 (1963), no. 2, 130-141.

[23] Jonathan C Mattingly, Andrew M Stuart, and Desmond J Higham, Ergodicity for sdes and approximations: locally lipschitz vector fields and degenerate noise, Stochastic processes and their applications 101 (2002), no. 2, 185-232.

[24] Jonathan C Mattingly, Andrew M Stuart, and Michael V Tretyakov, Convergence of numerical time-averaging and stationary measures via poisson equations, SIAM Journal on Numerical Analysis 48 (2010), no. 2, 552-577.

[25] Sean P Meyn and Richard L Tweedie, Markov chains and stochastic stability, Springer Science \& Business Media, 2012.

[26] J Náprstek and R Král, Multi-dimensional fokker-planck equation analysis using the modified finite element method, Journal of Physics: Conference Series, vol. 744, IOP Publishing, 2016, p. 012177.

[27] Bernt Øksendal, Stochastic differential equations, Stochastic differential equations, Springer, 2003, pp. 65-84.

[28] Hannes Risken, Fokker-planck equation, The Fokker-Planck Equation, Springer, 1996, pp. 6395.

[29] Christian P Robert, Monte carlo methods, Wiley Online Library, 2004.

[30] Otto E Rössler, An equation for continuous chaos, Physics Letters A 57 (1976), no. 5, 397-398.

[31] Yifei Sun and Mrinal Kumar, A numerical solver for high dimensional transient fokker-planck equation in modeling polymeric fluids, Journal of Computational Physics 289 (2015), 149-168.

[32] Surya T Tokdar and Robert E Kass, Importance sampling: a review, Wiley Interdisciplinary Reviews: Computational Statistics 2 (2010), no. 1, 54-60.

[33] Zixuan Wang, Qi Tang, Wei Guo, and Yingda Cheng, Sparse grid discontinuous galerkin methods for high-dimensional elliptic equations, Journal of Computational Physics 314 (2016), 244-263.

[34] EC Zeeman, Stability of dynamical systems, Nonlinearity 1 (1988), no. 1, 115. 
Yao Li: Department of Mathematics and Statistics, University of Massachusetts AMHERST, USA

E-mail address: yaoli@math.umass.edu 